\title{
A FREQUÊNCIA dE DISFAGIA EM IDOSOS EM UM hOSPITAL DA CIDADE DE CURITIBA - PR
}

\author{
The frequency of dysphagia in the elderly in a \\ hospital of the city of Curitiba - state of Paraná
}

Andréia Maneira ${ }^{1}$, Isabel de Lima Zanata²

1. Fonoaudióloga. Residente do Programa de Residência Multiprofissional em Saúde do Idoso. Fundação Estatal de Atenção em Saúde de Curitiba (FEAES)

2. Fonoaudióloga. Mestre em Distúrbios da Comunicação pela Universidade Tuiuti do Paraná, Especialista em Gerontologia pela Sociedade Brasileira de Geriatria e Gerontologia, Coordenadora da Residência Multiprofissional em Saúde do Idoso da Fundação Estatal de Atenção em Saúde de Curitiba (FEAES). ORCID: orcid.org/0000-0002-0902-5584

CONTATO: Andréia Maneira | Rua Casemiro Synderski, 37 | Rio Bonito | Irati | Paraná | Brasil | E-mail: andreiamaneira@hotmail.com

COMO CITAR Maneira A, Zanata IL. A frequência de disfagia em idosos em um hospital da cidade de Curitiba-PR. R. Saúde Públ. 2018 Jul.,1(1):20-26.

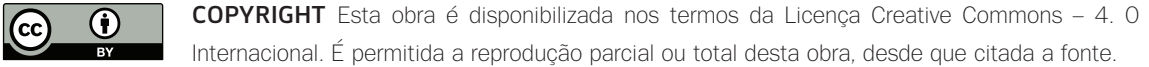

RESUMO O trabalho consistiu em analisar o perfil epidemiológico dos pacientes internados em um hospital na cidade de Curitiba - PR, durante um período de seis meses e identificar a frequência de disfagia nesta população. Tratase de uma pesquisa epidemiológica retrospectiva, descritiva a partir de análise de prontuário eletrônico. Os dados foram submetidos à análise descritiva. Sendo identificados 216 pacientes atendidos pelo serviço de fonoaudiologia da instituição, a amostra se configurou com média de idade igual a 83 anos e composta por 131 pacientes do sexo feminino e 85 do sexo masculino. A Hipertensão Arterial Sistêmica foi a doença com maior frequência nos idosos pesquisados e par a comorbidade a Desnutrição. 0 trabalho constatou que em relação às dificuldades de deglutição a disfagia de grau leve ocorreu com maior frequência e que há grande frequência de distúrbios de deglutição em idosos hospitalizados acometidos por doenças crônicas relacionadas ao envelhecimento.

PALAVRAS-ChAVE: Idoso. Transtornos de deglutição. Fonoaudiologia. Serviços de saúde para idosos. Epidemiologia. 
ABSTRACT The objective of the study was to analyze the epidemiological profile of patients admitted in a hospital in the city of Curitiba, state of Paraná, Brazil, for six months, and to identify the frequency of dysphagia among this population. It is a retrospective, descriptive epidemiological survey made with the electronic medical record analysis. Data were submitted to descriptive analysis. Among 216 patients being treated by the speech therapy service of the institution, the sample was configured with a mean age of 83 years, and consisted of 131 female patients and 85 male patients. Systemic Arterial Hypertension was the disease with the highest frequency in the elderly studied, and the comorbidity was Malnutrition. The study found that regarding swallowing difficulties, mild dysphagia occurred more frequently and there is a high frequency of swallowing disorders in hospitalized elderly patients with chronic aging-related diseases.

KEYWORDS: Aged. Deglutition disorders. Speech, Language and Hearing Sciences. Health Services for the Aged; Epidemiology.

\section{INTRODUÇÃO}

$\mathbf{N}$

os últimos anos, o Brasil vem apresentando um novo padrão demográfico que é caracterizado por redução de taxa de crescimento populacional e transformações na composição de sua estrutura etária, com um significativo aumento do contingente de idosos. Estas modificações têm imprimido importantes mudanças no perfil epidemiológico da população. ${ }^{1}$

O Brasil possui a quinta maior população idosa do mundo, com cerca de 28 milhões de pessoas com 60 anos ou mais. Atualmente, a proporção de pessoas idosas no país alcançou $13,7 \%$ da população geral, sendo o grupo de idosos longevos, aqueles que vivem 80 anos ou mais, a parcela que mais

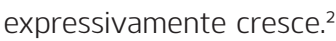

O resultado dessa dinâmica é a maior procura dos idosos por serviços de saúde. As internações hospitalares são mais frequentes e o tempo de ocupação do leito é maior quando comparado a outras faixas etárias. Dessa forma, o envelhecimento populacional se traduz em maior carga de doenças na população, mais incapacidades e aumento do uso dos serviços de saúde. ${ }^{3}$

O processo de envelhecimento pode ser definido como natural, progressivo, degenerativo, universal e intrínseco, caracterizado por menor eficiência funcional, com enfraquecimento dos mecanismos de defesa frente às variações ambientais e perda das reservas funcionais. ${ }^{4}$

Durante o envelhecimento ocorrem mudanças fisiológicas que acarretam dificuldades de deglutição. As alterações da deglutição podem ser originadas de causas primárias, como as decorrentes do envelhecimento, e secundárias, que seriam derivadas de agentes farmacológicos, condições de saúde e condições neurológicas que afetam essa população. $^{5}$

A avaliação clínica da deglutição é segura, não invasiva, tem baixo custo, é rápida, reproduzível e consome poucos recursos.

A disfagia reflete em problemas envolvendo a cavidade oral, faringe, esôfago ou transição 
esofagogástrica. A disfagia ou dificuldade na deglutição pode resultar na entrada de alimento na via aérea, resultando em tosse, sufocação/asfixia, problemas pulmonares e aspiração. Também gera déficits nutricionais, desidratação com resultado em perda de peso, pneumonia e morte. ${ }^{6}$

Em um ambiente hospitalar composto por idosos existe a presença de um elevado número de queixas referentes às dificuldades na deglutição. Parte refere-se às deglutições já adaptadas, outros às alterações mecânicas e estas dificuldades fazem da avaliação fonoaudiológica uma intervenção diferenciada para determinação da origem das dificuldades de deglutição. I d e n t i f i c a ç õ e s precoces merecem atenção, pois colaboram com a prevenção de agravos à saúde do idoso.

Conhecer sobre a epidemiologia da disfagia na população idosa proporciona o conhecimento dos fatores que determinam o problema, bem como auxilia o melhor entendimento das consequências relativas à saúde.

No atendimento ao paciente disfágico e em ambiente hospitalar, compete ao fonoaudiólogo colaborar juntamente com a equipe interdisciplinar na melhora clínica, buscando a redução do tempo de uso das vias alternativas de alimentação, o tempo de hospi talização e contribuição na melhoria do quadro pulmonar.

O presente trabalho teve por objetivo a identificação do perfil epidemiológico e a frequência de disfagia em idosos atendidos na instituição

\section{MATERIAL E MÉTODO}

O presente estudo epidemiológico, descritivo e retrospectivo foi realizado por análise documental em prontuário médico. O trabalho foi aprovado pelo Comitê de Ética em Pesquisa da Faculdade Pequeno Príncipe sob o parecer de número 2.113.89.

A coleta dos dados foi realizada por um único pesquisador, e optou-se por trabalhar com os prontuários eletrônicos de pacientes internados durante o período de junho a novembro de 2016 , selecionados os pacientes com idade maior que 65 anos e que passaram por avaliação fonoaudiológica durante o internamento, excluindo os pacientes que passaram pelo atendimento em unidade de terapia.

Desta forma utilizou-se 216 prontuários de pacientes atendidos na unidade de internação, 131 do sexo feminino e 85 do sexo masculino, com média de idade de 83,7 anos e com intervalo etário de 65 a 102 anos.

A análise dos dados buscou identificar o paciente em idade e sexo, aspectos da avaliação médica em doenças de base e comorbidades, aspectos da avaliação fonoaudiológica em presença e grau de disfagia.

Assegurando-se que todos os cuidados para garantir o sigilo, anonimato e privacidade dos participantes quanto aos dados confidencias foram realizados.

As variáveis epidemiológicas e clínicas foram analisadas de forma descritiva por meio de valores percentuais, média, e apresentados em forma de gráficos, utilizando o software Microsoft Office Excel.

\section{RESULTADOS}

Os resultados demonstram que a população estudada apresentou o número de doenças superior a 1, as mais evidentes foram Hipertensão Arterial Sistólica, Diabetes, Demência e Doença Cerebrovascular (Tabela 1). Para comorbidades a mais frequente no estudo é a Desnutrição (Tabela 2), e o tabagismo destacou-se como maior repetição nos hábitos de vida pregressa.

Na avaliação fonoaudiológica, os resultados nos mostram que $7,87 \% \quad(n=17)$ dos pacientes apresentaram deglutição funcional, 51,85 \% ( $n=112)$ disfagia leve, 16,67\% ( $n=36)$ disfagia moderada e 23,61\% ( $n=51$ ) apresentaram disfagia grave (Gráfico 1). 
Gráfico 1. Padrão de deglutição por e divisão por sexo.

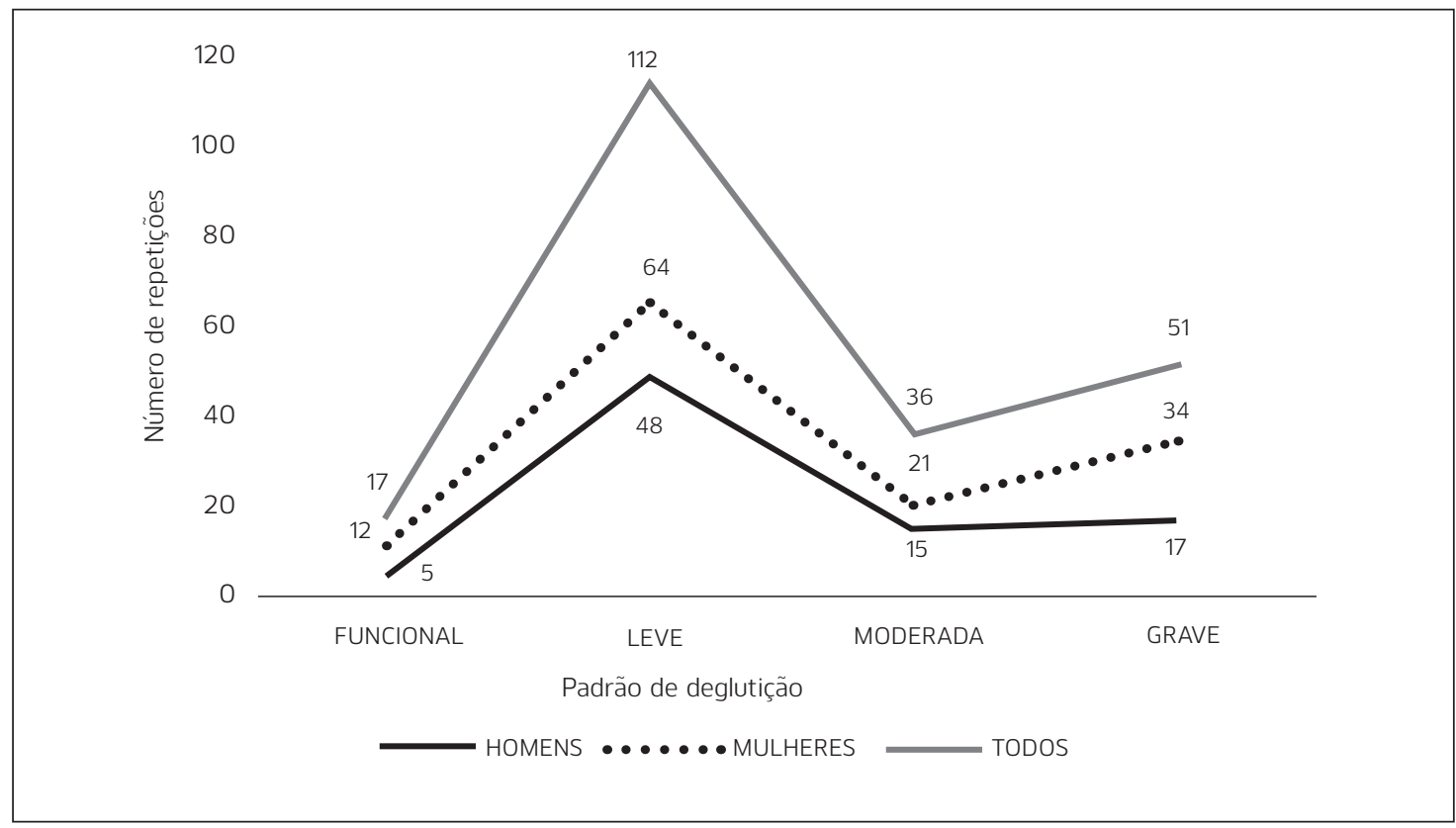

Gráfico 01: número de ocorrências nos pacientes por sexo e total.

Fonte: Elaborado pelos autores (2018)

O sexo feminino se configurou com os maiores números em relação ao sexo masculino.

Em relação à disfagia na unidade amostral segregada por sexo, configurou-se uma maior prevalência no sexo masculino sendo que acometeu 80 pacientes ( $n=85$ ), representando $94,11 \%$ dos casos deste sexo. Já no sexo feminino foram acometidas 119 idosas ( $n=131)$, resultando 90,83\% dos casos.
As tabelas a seguir indicam o percentual do padrão de deglutição em relação às doenças de maior frequência.

Em escala decrescente de porcentagem a Hipertensão sistólica tem o maior percentual de frequência com 66,67 \% e a Neoplasia o menor com $10,65 \%$.

Tabela 1. Porcentagem do padrão de deglutição por doença

\begin{tabular}{l|ccccc}
$\begin{array}{l}\text { DOENÇA/ PADRÃO } \\
\text { DE DEGLUTIÇÃO }\end{array}$ & FUNCIONAL & LEVE & MODERADA & GRAVE & $\%$ \\
\hline HAS & 14 & 75 & 24 & 31 & $66,67 \%$ \\
DM & 6 & 31 & 7 & 6 & $23,15 \%$ \\
DEMÊNCIA & 2 & 31 & 15 & 19 & $31,02 \%$ \\
DOENÇA & 1 & 26 & 17 & 13 & $26,39 \%$ \\
CEREBROVASCULAR & 3 & 17 & 1 & 2 & $10,65 \%$ \\
NEOPLASIA & 3 & & & & \\
\hline
\end{tabular}

Tabela 01: número de ocorrências nos pacientes e porcentagem segregados por doença em relação ao padrão de deglutição. Fonte: Elaborado pelos autores (2018) 
Tabela 2. Porcentagem do padrão de deglutição por comorbidade.

\begin{tabular}{l|ccccc}
$\begin{array}{l}\text { COMORBIDADE/ PADRÃO } \\
\text { DE DEGLUTIÇÃO }\end{array}$ & FUNCIONAL & LEVE & MODERADA & GRAVE & $\%$ \\
\hline HISTÓRICO TABAGISMO & 3 & 27 & 6 & 14 & $23,15 \%$ \\
HISTÓRICO ETILISMO & 1 & 7 & 3 & 5 & $7,41 \%$ \\
DESNUTRIÇÃO & 3 & 61 & 25 & 37 & $58,33 \%$ \\
\hline
\end{tabular}

Tabela 02: número de ocorrências nos pacientes e porcentagem segregados por comorbidade em relação ao padrão de deglutição. Desnutrição com 58,33 \% é a comorbidade com maior frequência.

Fonte: Elaborado pelos autores (2018)

\section{DISCUSSÃO}

No internamento hospitalar o idoso está exposto a complicações e a infecções, e ainda representa fator de risco para a disfagia. ${ }^{7}$

A deglutição envolve a participação de órgãos, músculos, nervos e depende da integridade dos mesmos para ocorrer de forma coordenada e eficiente, ${ }^{8}$ a desorganização do sistema estomatognático pode sofrer alterações decorrentes do envelhecimento causando a disfagia.

A presbifagia se caracteriza pelas modificações na função da deglutição durante o envelhecimento do ser humano, que desencadeia uma adaptação ao processo de alimentação e deixa o idoso propenso a desenvolver disfagia.

As características da deglutição em idosos são diversas e incluem dificuldades de controle e ingestão do bolo, diminuição da força mastigatória, acúmulo de resíduos, redução do grau de elevação da laringe, necessidade de deglutições múltiplas, maior ocorrência de tosse, maior probabilidade de aspiração após a deglutição e aumento da duração da fase esofágica. ${ }^{9}$

As dificuldades de deglutição acometem 16\% a $22 \%$ da população acima de 50 anos, alcançando índices de 70\% a 90\% de distúrbios de deglutição nas populações mais idosas ${ }^{10}$. Este estudo corrobora com o perfil epidemiológico observado, pois os resultados refletem que a disfagia estava presente em 90\% nos pacientes com média de idade de 83 anos.
Dentre os prontuários estudados foram encontrados 131 pacientes do sexo feminino, sendo a maioria do espaço amostral, este dado confirma a feminização da velhice no Brasil.

Feminização, que se explica, em parte, pela maior expectativa de vida das mulheres, associada aos fatores como: menor consumo de álcool e tabaco e diferenças de atitude em relação às patologias ${ }^{3}$. Este fato se reflete também pelo autocuidado ao identificar sinais e sintomas precoces.

Constatou-se que grande parte dos idosos é acometida por uma ou mais doenças crônicas, enfermidades que aumentam a frequência de internação hospitalar.

As doenças crônicas não transmissíveis, naturais do processo de envelhecimento são consideradas um sério problema de saúde coletiva, devido aos agravos que podem propiciar a ocorrência de outros agravos à saúde. As quatro principais são: doenças cardiovasculares, neoplasias, doenças respiratórias crônicas e diabetes ${ }^{11}$.

O estudo constata que essas doenças são rotineiramente diagnosticadas na população idosa. Identifica-se um número dentro do espaço amostral $(n=216)$ a amostragem de 144 pacientes com diagnóstico de Hipertensão Arterial e 50 pacientes com Diabetes ou ambas.

No Brasil, a HAS afeta mais de 30 milhões de brasileiros (36\% dos homens adultos e 30\% das mulheres) e é o maior fator de risco para o desenvolvimento das doenças cardiovasculares, com destaque para o acidente vascular encefálico 
(AVC) e o infarto do miocárdio, as duas maiores causas isoladas de mortes no país. ${ }^{12}$

A Diabetes Mellitus (DM) é uma disfunção metabólica caracterizada pela presença da hiperglicemia e pode ser resultado de defeitos, tanto na ação quanto na secreção da insulina, assim como em ambos. Um estudo indica que a prevalência da DM varia de 7 a 14\% na população acima de 30 a 69 anos. $^{13}$

O percentual para a disfagia encontrado em pacientes com Hipertensão Arterial foi frequente em 90,27\% e para a Diabetes foi em 88\% dos casos do estudo.

Um estudo realizado anteriormente avaliou a deglutição observando o tempo de trânsito faríngeo durante a deglutição de líquido. Participaram desse estudo 63 idosos divididos em dois grupos, sendo um saudável e outro com doenças frequentemente encontradas nessa população (hipertensão arterial, osteoartrite, diabetes mellitus, doença da tireoide, hipercolesterolemia, doença do refluxo gastroesofágico e depressão). Os autores verificaram aumento significativo do tempo de trânsito faríngeo para os idosos com doenças sistêmicas, especialmente no grupo com hipertensão arterial. ${ }^{14}$ Concluíram que a presença de doenças comuns à população idosa estava associada à deterioração da função da deglutição, não podendo tal alteração ser considerada decorrente somente do envelhecimento.

Quanto ao histórico de neoplasias, 23 (n=216) dos pacientes apresentaram a doença, descritas em seu prontuário. Segundo o Instituto Nacional de Câncer José de Alencar, em 2013 as estimativas de câncer no Brasil foram de 518.510 novos casos da doença.

No que se refere ao número de pacientes com sintomas de disfagia, ocorreram 86,96 \%, não diferenciados por especificidade da neoplasia.

Sobre a frequência de Demência de acordo com o estudo, 31,6\% dos pacientes possuíam o diagnóstico em prontuário.

Em estudo15 sobre demência constatou-se que pacientes demenciados e com outras doenças neurológicas podem apresentar comprometimento funcional e cognitivo por um longo período antes da morte e também desenvolver disfagia, desnutrição, pneumonia e imobilidade, tornando-se parcial ou totalmente dependentes para as atividades da vida diária.

A demência inclui como sintoma da evolução da doença, a disfagia 16, no presente trabalho a afirmação é demonstrada pela presença em 67 idosos com quadro demencial. Deste valor 97\% dos casos apresentam dificuldades de deglutição.

Em pesquisa realizada em idosos com demência, foi observado que a maioria desses idosos apresentou dificuldades durante a alimentação ou em atividades envolvidas nesta função, como o preparo do alimento, o manuseio de utensílios e a higiene oral, dependendo da assistência de um cuidador. Com isso, 16 concluiu que os idosos com demência precisam de acompanhamento fonoaudiológico e nutricional periódico, a fim de minimizar suas limitações e incapacidades relacionadas à alimentação, prevenindo a desnutrição, a desidratação e outras complicações.

Destaca-se significância no estudo para as doenças cerebrovasculares, as quais têm etiologia multifatorial, e dentre os prontuários pesquisados foram encontrados 50 pacientes com histórico de Acidente Vascular Cerebral (AVC) ou Aneurisma.

A incidência de disfagia pós-AVC pode variar de 42 a $67 \%$ e a sua presença está associada a um aumento do risco de complicações pulmonares por aspiração de saliva e/ou alimento, desnutrição, desidratação, hospitalização prolongada e morte. 17,18

Segundo o presente estudo, encontrou-se 98\% de pacientes com doenças cerebrovasculares e sintomas de disfagia, número significativamente maior que o do estudo acima citado.

Foram encontrados 126 pacientes com estado nutricional comprometido. A disfagia foi frequente em $98 \%$ dos casos pesquisados.

A disfagia pode levar à desnutrição e à desidratação por inadequação dietética e em razão da consistência dos alimentos:19

A desnutrição é um problema comum nas pessoas idosas, que é raramente reconhecido e diagnosticado, levando insucesso e incapacidade 
para cobrir as necessidades nutricionais dos doentes. Apesar dos estudos apontarem diferentes critérios para identificar a má nutrição nos idosos, há clara evidência que a desnutrição afeta pessoas que vivem nas suas casas, institucionalizadas e em maior extensão os pacientes com doenças agudas ou crônicas, que se encontram hospitalizados ${ }^{20}$.

Na pesquisa percebe-se a grande prevalência de desnutrição em ambiente hospitalar. principalmente pela população estudada ser composta por idosos.

A intervenção precoce para o diagnóstico da disfagia contribui para melhores resultados e a melhora do idoso hospitalizado, principalmente em pacientes com doenças crônicas que apresentam como complicação a disfagia. ${ }^{21}$

\section{CONCLUSÕES}

O desenvolvimento do presente trabalho oportunizou a identificação epidemiológica da população estudada e a frequência de disfagia.

Constatou-se grande incidência de doenças crônicas nos indivíduos da pesquisa, causando internamentos hospitalares frequentes. Confirmouse também que há grande frequência de disfagia nos idosos internados na instituição.

O diagnóstico da disfagia merece grande atenção devido às implicações clínicas causadas pelo sintoma.

\section{REFERÊNCIAS}

1. Instituto Brasileiro de Geografia e Estatística. Indicadores Sociodemográficos e de Saúde no Brasil 2009. Disponível em: ww2.ibge.gov.br

2. Ministério da Saúde. Conjunto de ações foca na saúde do idoso. Disponivel em: www. Brasil.gov.br.

3. Veras R. Envelhecimento populacional contemporâneo: demandas, desafios e inovações. Saúde Pública 2009. 43(3). 548554

4. Ribeiro A. Aspectos biológicos do envelhecimento. In: RUSSO IP. Intervenção Fonoaudiológica na Terceira Idade. Rio de Janeiro: Revinter, 2004.

5. Jaradesh S. Neurophysiology of swallowing in the age. Dysphagia. 1994; 9:218-20
6. Padovani AR, Moraes DP, Mangilli LD, Andrade CRF. Protocolo de Avaliação do Risco para a Disfagia (PARD). In: Andrade CRF, Limongi SCO (Org). Disfagia: prática baseada em evidências. São Paulo: Sarvier; 2012. p. 62-73.

7. Guerra IC, Ramos OS, Cerqueira A. Risco de hospitalizações repetidas em idosos usuários de um centro de saúde escola. Cad Saúde Pública. 2007; 23(3): 585-92.

8. Kikawada M, Iwamoto T. Takasaki M. Aspiration and infection in the elderly. Drugs Aging. 2005; 22(2):115-30.

9. Nilsson $H$, Ekberg O, Olsson R, Hindfelt B. Quantitative aspects of swallowing in an elderly nondysphagic population. Dysphagia.1996:11(3):180-4.

10. Santoro PP. Disfagia orofaríngea: panorama atual, epidemiologia, opções terapêuticas e perspectivas futuras. Revista CEFAC. 2008;10(2)

11. Schmidt MI, Duncan BB, Silva GA, Menezes AM, Monteiro CA: Barreto SM. Chronic non-communicable diseases in Brazil: burden and current challenges. Lancet. 2011;377 (9781):1949-61.

12. VI Diretrizes Brasileiras de Hipertensão. Arq Bras Cardiol. 2010:95

13. Lyra R, Silva RS, Montenegro JRM, Matos MVC; Cézar NJB, Da Silva ML. Prevalência de diabetes melitos e fatores associados em população urbana adulta de baixa escolaridade e renda do sertão nordestino brasileiro. Arq Bras Endocrinol Metab. 2010:54(6):5606

14. Kendall KA: Leonard RJ: McKenzie S. Common medical conditions in the elderly: impact on pharyngeal bolus transit. Dysphagia. 2004:19(2):71-7.

15. Palecek EJ, Teno JM, Casarett DJ. Hanson LC, Rhodes RL, Mitchell SL. Comfort feeding only: a proposal to bring clarity to decision-making regarding difficulty with eating for persons with advanced dementia. J Am Geriatr Soc. 2010;58(3):580-4

16. Goes VF, Mello-Carpes PB, Oliveira LO, Hack J, Magro M, Bonini JS. Avaliação do risco de disfagia, estado nutricional e ingestão calórica em idosos com Alzheimer. Rev. Latino-Am. Enfermagem mar.-abr. 2014;22(2):317-24

17. Sanches EP, Bilton T, Ramos LR. Análise descritiva da alimentação de idosos com demência. Distúrb Comun. 2000: 11(2):227-49.

18. Itaquy RB: Favero SR: Ribeiro MC: Barea LM: Almeida ST. Mancopes R. Disfagia e acidente vascular cerebral: relação entre o grau de severidade e o nível de comprometimento neurológico. J Soc Bras Fonoaudiologia. 2011; 23(4):385-9.

19. | Consenso Brasileiro de Nutrição e Disfagia em Idosos Hospitalizados. Barueri, SP: Minha Editora, 2011.

20. Oliveira MM. Terapia Nutricional e Disfagia: A Importância do Acompanhamento Nutricional. Rev Brasileira de Ciências da Saúde, ano 6, n 16, abr/jun 2008

21. De Paiva KM, Xavier IC, Farias N. Envelhecimento e Disfagia: Uma questão de saúde pública. Aging and Dysphagia: a Public Health Issue. Journal of Aging \& Inovation, 1 (6): 67-77, 2012

RECEBIDO: $27 / 02 / 2018$ 